\title{
Une démarche d'étude agronomique des climats naturels; le cas de différences d'altitude
}

\author{
M Lafarge \\ INRA, station d'agronomie, 12 avenue du Brézet, 63039 Clermont-Ferrand, France
}

(Reçu le 8 août 1990; accepté le 7 mars 1991)

\begin{abstract}
Résumé - Ce texte présente l'intérêt et les limites des différentes démarches d'étude des climats naturels : expérimentation, simulation et enquête éventuellement associée à un dispositif expérimental. Une démarche hybride entre enquête et expérimentation paraît particulièrement bien adaptée à l'analyse des effets de différences d'altitude. On décrit ensuite le dispositif et le suivi de végétation mis en œuvre pour comparer la croissance, le tallage et la formation de la fertilité d'épi d'une orge de printemps dans différents sites du Massif central. Entre ces situations culturales écologiquement contrastées, les mesures révèlent des différences sensibles de variabilité. L'emploi d'une méthode d'analyse de variance traitant correctement les dispositifs non orthogonaux est nécessaire dans ce type d'étude. Les rendements obtenus en montagne peuvent égaler ceux de plaine. Les effets du climat s'observent sur les états successifs de la végétation.
\end{abstract}

méthodologie / effet agronomique du climat / dispositif multilocal / altitude / céréale

Summary - An approach for agronomical investigation of natural climates; the case of differences in altitude. This paper reviews the interest and the limitations of different approaches in the study of the agronomical effects of natural climates: experimentation, simulation and enquiry, possibly associated with an experimental design (fig 1). Such a "hybrid approach" between enquiry and experimentation appears particularly well adapted to the agronomical analysis of differences in altitude. This paper describes an experimental design (table I) and vegetation survey implemented to compare growth, tillering and spike fertility formation of a spring barley in several sites of the French Massif Central (fig 2). Related to the phenological phases of the crops, the climates were very different (fig 3). Incident radiation per degree-day (table II) and water availability in the upper layer of the soil (table III) explain some of the results. Yields can be similar at mountain sites and at low altitude (fig 4), but yield components differ (table IV). Successive states of vegetation show effects of climate. The data reveal sizeable differences in variability between situations (table V). Such a type of study requires a method of analysis of variance which processes non orthogonal designs adequately.

methodology / agronomical effect of climate / multilocal design / altitude / cereal

\section{INTRODUCTION}

Cet article présente d'un point de vue méthodologique la démarche et le dispositif choisis pour examiner les effets climatiques de l'altitude sur la croissance et le développement des céréales dans le Massif central français. L'étude devait permettre de proposer aux éleveurs montagnards des références pour la conduite de leurs céréales. Elle visait plus fondamentalement une meilleure connaissance des réactions, en peuplements réels, du tallage, de la fertilité d'épi et de la croissance à des climats plus contrastés qu'en zone de grande culture.

\section{LES DÉMARCHES D'ÉTUDE DES EFFETS DU CLIMAT}

On sait depuis l'antiquité que le climat détermine le cours de la végétation et la récolte utile. Le caractère incontrôlable des événements climatiques que subit une culture au champ s'oppose pourtant à leur étude expérimentale directe dans les conditions naturelles. Plusieurs attitudes sont possibles face à cet obstacle :

- simplifier le climat naturel de telle sorte que les variantes participant à l'étude puissent être considérées comme les modalités d'un facteur contrôlé; on peut alors mettre en œuvre une démarche expérimentale; 
- simuler la réponse d'une culture à des effets connus d'éléments du climat et comparer le comportement simulé au comportement réel de la végétation;

- enquêter, c'est-à-dire relier des effets observés à des causes identifiées a posteriori (Gras et al, 1989).

De nombreuses études d'effets climatiques peuvent être examinées du point de vue de l'intérêt et des limites de la démarche dont elles procèdent.

\section{Expérimentation}

En expérimentation au champ, on traduit aisément le climat en modalités d'un facteur contrôlé en répétant un dispositif cultural plusieurs années en un même lieu, ou en plusieurs lieux s'ils sont assez distincts (Ellis et Brown, 1986). Le facteur "année" évite le biais de différences de sol, mais le climat n'est jamais défini que globalement dans ces expériences. Un effet significatif ne fournit pas d'informations sur la façon dont il a agi (Fuchs, 1984), même si l'on met en œuvre des analyses multivariables modernes (Riggs, 1986). On peut seulement le quantifier relativement à la moyenne générale, année par année, dans le cadre d'un modèle d'analyse de variance (Rellier, 1980).

Toujours au champ, certaines composantes du climat naturel peuvent être modifiées par une intervention technique. II apparaît alors des modalités des conditions de milieu induites par l'intervention (ou par son absence) auxquelles on peut rapporter les différences observées sur la végétation. Quand on a irrigué, on traite d'effets de sécheresse (Lawlor et al, 1981). Il s'agit alors de l'ensemble indistinct des facteurs induits par la sécheresse. Sans éléments extérieurs d'interprétation ni observations contemporaines du fonctionnement des écosystèmes, les résultats ne peuvent pas en effet être attribués à des défauts de transpiration (frein aux échanges gazeux) ou de capture de l'azote en sol sec.

Les conditions contrôlées permettent d'expérimenter finement sur des éléments de climat, mais l'artificialisation pose des problèmes de signification de ces résultats pour les situations réelles. Evans (1963) considère que la non reproduction des microhétérogénéités spatiotemporelles de l'environnement naturel des plantes modifie les résultats, même au niveau de phénomènes physiologiques.

\section{Simulation}

La simulation éprouve différentes synthèses d'études expérimentales sectorielles. Un modèle fonctionnellement pertinent et valide dans toutes les conditions naturelles pourrait être perçu comme le résultat d'une connaissance complète des effets agronomiques du climat. C'est pourquoi de nombreux travaux suivent cette voie; parmi les plus récents en Europe on peut citer Cabelguenne et al (1988) et Van Diepen et al (1989). Les résultats publiés sont toujours satisfaisants, sans pour autant épuiser le sujet.

Le fonctionnement des végétations en place met en jeu des phénomènes écologiques et morphologiques qui ne sont pas pris en compte dans les processus modélisés. On surmonte cet obstacle en prévoyant l'introduction de paramètres qui caractérisent empiriquement la culture étudiée, c'est-à-dire indistinctement l'espèce et les modalités des phénomènes écologiques et morphologiques la concernant dans les conditions où elle est placée.

Dans le modèle de Cabelguenne et al (1988), des indices de sensibilité aux stress, l'indice foliaire maximal, la hauteur maximale des plantes et l'indice de récolte (rapport grains/poids sec des parties aériennes) sont des données par espèce et système de culture (Charpenteau et al, 1986; Cabelguenne et al, 1988). Des résultats gravement divergents entre simulation et réalité apparaissent certaines années où les valeurs données à ces paramètres sont erronées (Cabelguenne et al, 1986). Leur indépendance vis-à-vis du climat est pour le moins constestable, mais la construction de sous-modèles les simulant supposerait une meilleure connaissance de l'influence du climat naturel sur des processus écologiques difficiles à étudier expérimentalement.

\section{Enquête et démarche hybride}

Le climat naturel ne se prête bien à étude directe que par enquête, mais les connaissances qu'on en retire dépendent des méthodes mises en œuvre. L'ajustement par régressions d'un paquet de données multilocales (Hough, 1975) ne donne pas plus d'informations généralisables que l'effet année d'une expérimentation pluriannuelle. On obtient plus de renseignements quand on peut relier par tables de contingence des fréquences de caractères climatiques par périodes à des fréquences par classes de rendement (Stegemann et al, 1987). Les informations obte- 
nues peuvent être aussi précises qu'à l'issue d'une étude expérimentale fine quand on pratique un suivi et des mesures détaillées, comme dans l'étude Barley and its environment (Biscoe et al, 1975; Gallagher et al, 1976).

Cependant, dès qu'on veut étudier les effets du climat naturel en comparant des cultures en situations différentes, l'enquête ne permet pas de supposer égales par ailleurs les conditions non étudiées. On peut résoudre en partie cette difficulté en adoptant une démarche hybride (Gras et al, 1989) entre enquête et expérimentation, du genre de celle mise en œuvre par Boiffin et Sébillotte (1975). Malgré leurs définitions, il n'y a pas en effet d'opposition formelle entre enquête et expérimentation, à partir du moment où l'on rapporte les caractères observés non pas directement aux interventions définissant le dispositif (fertilisation, dose de semis; fig 1a) mais aux états susceptibles d'agir effectivement sur la végétation (fig 1 b, c, d). En expérimentation pure, ces états (niveau d'alimentation minérale, peuplement) doivent dépendre directement des facteurs contrôlés par le dispositif (fig 1 b). En enquête, ils ne dépendent que de conditions non maîtrisées (fig $1 \mathrm{~d}$ ) dans la définition desquelles lintervention de l'observateur s'est limitée au choix des situations étudiées.

\section{CHOIX D'UNE DÉMARCHE POUR ÉTUDIER L'EFFET DES CLIMATS NATURELS D'ALTITUDE SUR UNE MÊME ESPÈCE}

Pour disposer des éléments d'une synthèse pertinente, l'analyse des effets agronomiques du climat doit concerner l'ensemble des aspects des

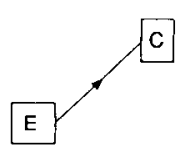

(a)

Expérimentation (empirique)

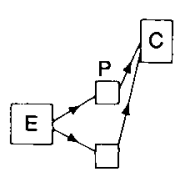

(b)

Expérimentation à tendance explicative

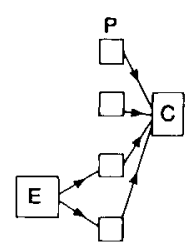

(c) hybride

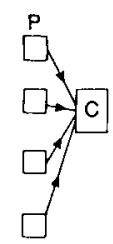

(d)
Fig 1. Relations causales envisagées dans 4 types de démarche d'études agronomiques (tirée de Gras et al, 1989). E: traitements expérimentaux; $C$ : caractères étudiés; $P$. "paramètres" (états du milieu, conditions) intervenant sur les caractères étudiés. Les "paramètres" sont les facteurs écologiques directs des caractères étudiés. On ne les considère pas en (a). En (b), on se limite à ceux qui sont directement modifiés par les traitements expérimentaux. En (c) on prend en compte, en plus, certains états du milieu indépendants des traitements expérimentaux. En (d), aucun des facteurs directs considérés n'a été modifié par une intervention expérimentale (d'après Gras et al, 1989). relations entre culture et milieu. Une voie intéressante est l'étude d'un même matériel végétal entre milieux naturels bien différenciés par le climat. La comparaison de climats naturels donne en effet une présomption de cohérence écologique aux résultats obtenus. Une différence d'altitude offre en plus des contrastes climatiques marqués à faible distance, ce qui facilite l'homogénéité des observations.

\section{Démarches pratiquées en études d'effets de différences d'altitudes}

Peu d'études comparant les mêmes cultivars à différentes altitudes ont été publiées. La plupart consistent en un dispositif expérimental multilocal, l'élévation au-dessus du niveau de la mer définissant chaque modalité du facteur altitude. Souvent, on cultive en chaque lieu sur le sol qu'on y trouve (Kobold, 1974; Chamberlin et Insomphun, 1982). Hunter et Grant (1971) donnent à l'altitude une signification plus purement climatique en installant leurs cultures dans des bacs remplis des mêmes terres dans tous les sites.

Dans la logique de ces dispositifs, l'effet de l'altitude est généralement simplement exprimé par les coefficients des régressions de différents caractères concernant la végétation sur l'élévation au-dessus du niveau de la mer. L'information qu'on peut retirer de ces études en matière d'action du milieu sur la culture est alors minime, quelle que soit l'ampleur et la rigueur du travail expérimental. Chamberlin et Insomphun (1982) n'ont calculé que des moyennes, mais ils ont fourni quelques éléments de discussion en enregistrant des composantes du rendement et le rayonnement global par phase de cycle dans chaque site.

Prince (1976) a adopté une autre démarche. II a comparé des suivis de croissance effectués dans ses différents sites d'étude sur des orges subissant aux mêmes stades des manipulations de surface assimilatrice ou de nombre de caryopses. II a aussi pratiqué des transferts de plantes entre sites à des stades déterminés. Les informations tirées de cette combinaison entre enquête et expérimentation sont riches et bien étayées.

\section{Les motifs du choix d'une démarche hybride}

Les études de différences d'altitude déjà publiées montrent qu'on ne peut analyser l'effet du climat qu'en pratiquant l'enquête, c'est-à-dire : 
- en recherchant a posteriori la meilleure relation entre des caractères de la végétation et des caractères climatiques contemporains enregistrés;

- en "suivant" la végétation, c'est-à-dire en calant les mesures et observations sur les phases successives du cycle dans chaque situation.

Parallèlement, les comparaisons du comportement de la végétation entre sites supposent que des conditions comme l'alimentation minérale soient bien définies partout, ce qui nécessite un dispositif expérimental. Si l'étude climatique doit répondre à une hypothèse, le choix des situations doit être structuré comme dans un plan d'expérience. Nous avons donc adopté pour notre propre étude (Lafarge, 1991) une démarche hybride entre enquête et expérimentation.

\section{ASPECTS EXPÉRIMENTAUX}

\section{Orientation de l'étude}

Nous avons choisi une céréale de printemps pour écarter les questions de passage de l'hiver, souvent traitées. L'orge a été retenue pour faciliter l'étude de la fertilité d'épi : elle a un épi non ramifié, avec une seule indétermination. Le décompte des ébauches florales avortées y est plus aisé et plus sûr que chez le blé (Kirby et Appleyard, 1981).

Dans les montagnes des régions tempérées, le tallage herbacé (Kobold, 1974) et la fertilité des épis (Lafarge et al, 1982; Lafarge, 1983) semblent limités. On peut traduire ces constatations contingentes en hypothèses générales raisonnables, à partir des conséquences climatiques d'une élévation en altitude :

- la baisse de la température moyenne entraîne un retard dans la sortie de l'hiver. La levée des céréales de printemps et la reprise de végétation des céréales d'automne se font en jours plus longs. Plus on s'élève en altitude, plus l'initiation florale et la montaison doivent suivre de près le démarrage de la croissance;

- le nombre d'ébauches d'épillet par épi est réduit, notamment chez l'orge, par une anticipation de l'intitiation florale (Kirby et Faris, 1970) et par la longueur du jour pendant leur formation (Paleg et Aspinall, 1966);

- l'émission des talles herbacées cesse quand l'azote ne suffit plus aux besoins (Aspinall, 1961; Masle-Meynard, 1981), mais le bilan s'apprécie au niveau de la plante. La montaison peut ainsi contribuer à l'arrêt du tallage : dans chaque plante, les tiges les plus précoces, aux besoins croissant rapidement avec leur montée (Vincent, 1986), sont servies prioritairement (Masle, 1985).

\section{Milieux utilisés et structure du dispositif expérimental multilocal}

Le dispositif expérimental repose sur l'utilisation de 3 sites INRA étagés en altitude en Auvergne (fig 2). Le premier, Malintrat, en plaine $(320 \mathrm{~m})$, est en zone de grande culture, sur terre noire argilo-calcaire (Collier, 1949). Le second, Saint Genès, est en moyenne montagne $(880 \mathrm{~m})$, dans une région herbagère autrefois cultivée (Bazin et Larrère, 1983) où la céréaliculture est actuellement possible sans difficultés particulières de récolte; il porte un sol d'un brun modérément acide, sur granite. Le troisième, Landeyrat, est à $1120 \mathrm{~m}$, en zone d'estive. II est au-dessus de la limite supérieure de culture des céréales, telle qu'on peut la définir par des risques acceptables

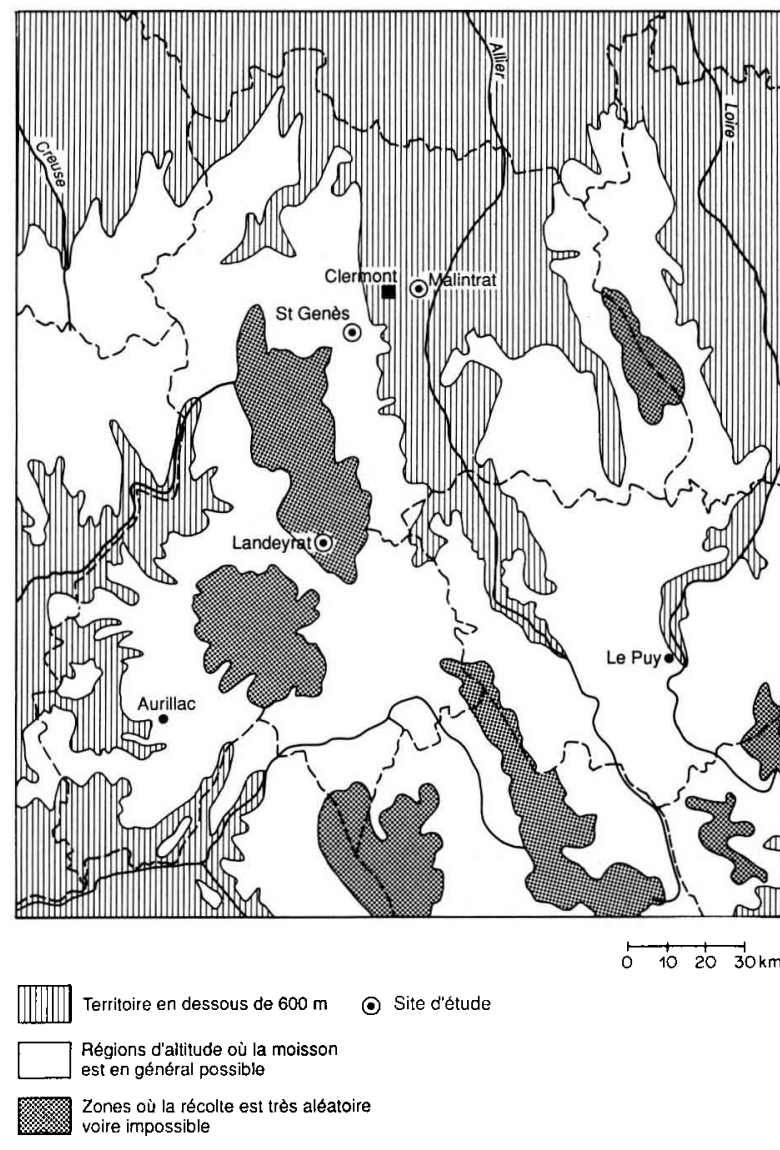

Fig 2. Localisation des sites d'étude par rapport à la zone d'altitude du Massif central où la récolte à la moissonneuse batteuse encourt des risques acceptables (carte tirée de Lafarge, 1987). 
pour un agriculteur de pertes de récolte par mauvais temps au moissonnage-battage (Lafarge, 1987). II porte un andosol développé sur basalte (Hétier, 1971). Les 2 sites de montagne avaient déjà été cultivés avec succès en orge dans les années antérieures.

Les limitations au tallage et à la fertilité des épis, qui constituent les hypothèses de base de l'étude des effets du climat, ne peuvent pas être étudiées au champ sous leur seul aspect morphogénétique en relation avec la photopériode. Elles dépendent aussi de conditions de croissance. L'altitude peut très bien n'être limitante que par la pauvreté nutritionnelle de son milieu.

Le dispositf comporte donc des traitements climatiques et des traitements culturaux. Dans les traitements climatiques, on tente de séparer la longueur du jour au démarrage d'autres facteurs, notamment la température et les conditions qui lui sont liées, en combinant sites et dates de semis. Dans chaque situation culturale créée par les traitements climatiques, on installe un jeu de conditions d'alimentation azotée et de concur- rence. Le dispositif d'ensemble est résumé au tableau $\mathrm{I}$.

\section{Les traitements du dispositif}

Le premier traitement climatique consiste à faire naître les plantes sous des longueurs croissantes de jour mais à des températures voisines. C'est le semis normal de sortie d'hiver en chaque lieu. Le second fait varier la température pour une naissance en jours longs $(15 \mathrm{~h}$ à notre latitude proche de $45^{\circ} \mathrm{N}$ ). II associe le semis normal du site le plus élevé à des semis plus tardifs aux altitudes inférieures.

$\mathrm{Au}$ niveau des traitements culturaux, nous cherchons à disposer d'un témoin peu limitant pour les caractères morphologiques sur lesquels portent les hypothèses, c'est-à-dire une basse densité de semis bien fertilisée. Nous avons choisi une densité moitié de celle qui se pratique habituellement en cultures productives en plaine. Pour l'azote, il fallait trouver un compro-

Tableau I. Caractères généraux du dispositif expérimental. Rappel des altitudes; Malintrat : $320 \mathrm{~m}$; Saint-Genès : $880 \mathrm{~m}$; Landeyrat : $1120 \mathrm{~m}$.

\begin{tabular}{|c|c|c|c|}
\hline \multicolumn{2}{|c|}{ Traitements climatiques } & \multicolumn{2}{|c|}{ Traitements culturaux } \\
\hline $\begin{array}{l}\text { Date de semis } \\
\text { (en année normale) }\end{array}$ & $\begin{array}{l}\text { Conséquences attendues } \\
\text { sur les conditions de } \\
\text { croissance-développement }\end{array}$ & Densité de semis & Dose d'azote \\
\hline $\begin{array}{l}\text { Semis normal } \\
\text { Fin février à Malintrat } \\
\text { Fin mars à St-Genès } \\
\text { Fin avril à Landeyrat }\end{array}$ & $\begin{array}{l}\text { Début de végétation dans } \\
\text { des conditions voisines de } \\
\text { température mais dans des } \\
\text { climats lumineux différents }\end{array}$ & $\begin{array}{l}2 \text { densités : } \\
\text { - une basse, identique } \\
\text { partout }\left(150 \text { grains } / \mathrm{m}^{2}\right) \\
\text { - une forte, croissante } \\
\text { avec les dates attendues } \\
\text { pour la montaison } \\
\left.\text { (300, } 400 \text { et } 650 \text { grains } / \mathrm{m}^{2}\right)\end{array}$ & $\begin{array}{l}2 \text { niveaux : } \\
\text { - néant } \\
\text { - apport fractionné } \\
\text { ajustant la ressource } \\
\text { à } 210 \mathrm{~N}\left({ }^{*}\right) \text { compte tenu } \\
\text { du reliquat } \\
\text { posthivernal }\end{array}$ \\
\hline $\begin{array}{l}\text { Semis tardifs } \\
\text { Début mai à St-Genès } \\
\text { Fin mai à Malintrat }\end{array}$ & $\begin{array}{l}\text { Début de montaison simultané } \\
\text { avec le semis normal de } \\
\text { Landeyrat : naissance dans } \\
\text { des conditions voisines } \\
\text { de photopériode puis } \\
\text { développement floral sous } \\
\text { le même éclairement mais } \\
\text { à des températures différentes }\end{array}$ & & \\
\hline
\end{tabular}

(") Objectifs des traitements culturaux repérés par une astérisque : - fortes densités variables : compensation par un peuplement dru des limitations attendues à la fertilité des épis et au tallage. - ressource azotées ajustée à 210 unités : nutrition la plus semblable possible peu limitante en début de végétation et permettant 70 q/ha de grain. 
mis entre une alimentation peu limitante, surtout lors du tallage et de la formation de l'épi avec des risques acceptables de verse. La moitié de la dose serait apportée peu après levée, et la fumure totale calculée à partir de la méthode de Rémy et Hébert (1977). L'objectif de rendement adopté est la production des meilleures cultures contemporaines d'orge de printemps en plaine, $70 \mathrm{q} / \mathrm{ha}$, jamais dépassée dans les 2 sites de montagne. Faute d'éléments sérieux de prévision pour tous les sites, on a considéré comme nul le bilan des termes minéralisation nette et reliquat à la moisson. Le reliquat posthivernal ayant été de $110 \mathrm{~N}$ a Saint-Genès et Malintrat, et de $50 \mathrm{~N}$ à Landeyrat, on a apporté $50+50$ dans les 2 premiers sites et $80+80$ à Landeyrat. Ce traitement témoin est dénommé BN.

Le dispositif cultural est complété par une même basse densité sans fumure azotée, qui fera apparaître la végétation permise par la fertilité locale. II se nomme BO. Le comportement des plantes dans les conditions d'une culture aussi productive que possible dans chaque condition climatique est également intéressant. Nous avons semé une forte densité, croissante en fonction des limitations les plus probables à la morphogenèse, c'est-à-dire celles qui découlent de la longueur du jour. Deux traitements FN et FO sont obtenus avec les mêmes modalités de fertilisation qu'en basse densité.

Les risques phytosanitaires les plus sérieux de chaque situation ont été limités. Pour les semis tardifs, le lit de semence a été traité en plein contre les mouches destructrices des apex (principalement des oscinies, dans nos conditions). À Landeyrat, la végétation a été traitée en montaison et à l'épiaison contre la rhynchosporiose et les cécydomies. Les traitements culturaux FO et FN y ont été répétés hors zone traitée.

Nous avons cultivé une seule variété, Bérénice. Toute la semence utilisée provient d'un même lot. Six répétitions d'une disposition en bloc ont été mises en place dans chaque site à chaque époque d'implantation. Un accident au semoir lors du semis tardif à Saint-Genès a entraîné l'abandon des blocs $4,5,6$. Quelques jours après, un dispositif de 3 blocs $(7,8,9)$ a été semé à quelques mètres de l'autre, mais sur un terrain hâtivement préparé. Les conditions sont suffisamment différentes entre ces 2 groupes de blocs pour qu'on doive les considérer comme 2 situations culturales distinctes.

\section{CARACTÉRISATION DES ÉTATS}

\section{Les états induits par les traitements culturaux}

Les densités de peuplement sont faciles à définir par des comptages. En début de végétation, on a aussi tenu compte des espaces vides de plus de $10 \mathrm{~cm}$ sur la ligne.

Le niveau d'alimentation azotée est beaucoup plus difficile à caractériser. Après discussion de la méthode des courbes de dilution de l'azote dans le poids sec des parties aériennes (Lemaire et Salette, 1984; Greenwood et al, 1986), nous l'avons adoptée pour l'explication des gains de poids sec (Lafarge, 1991). L'absence habituelle de tallage en pleine montaison malgré des teneurs souvent non limitantes selon cette méthode laisse penser que l'alimentation azotée doit être appréciée autrement pour la morphogenèse.

\section{Caractérisation du climat}

Les enregistrements obtenus pour chaque site sont transformés en variables climatiques par phase de cycle végétatif pour chaque situation culturale. La figure 3 donne une vue d'ensemble des conditions pour 3 variables importantes pour la croissance. Une expression des données calée sur le cycle végétatif montre les différences importantes de climats que nos cultures rencontrent. C'est aussi vrai pour l'énergie reçue, bien que le rayonnement solaire arrivant au sol soit rarement différent entre les 3 sites à une même date calendaire.

\section{Indicateur d'énergie disponible}

Le rayonnement solaire absorbé limite le gain de poids sec d'un couvert donné (Gosse et al, 1986) alors que la température limite la croissance en taille des végétaux (Durand, 1967). Sur une durée d'au moins quelques nycthémères, l'expression de l'intensité de rayonnement global relativement à une indexation thermique du temps peut être interprétée comme le rapport entre un potentiel d'assimilation du carbone et un poten- 


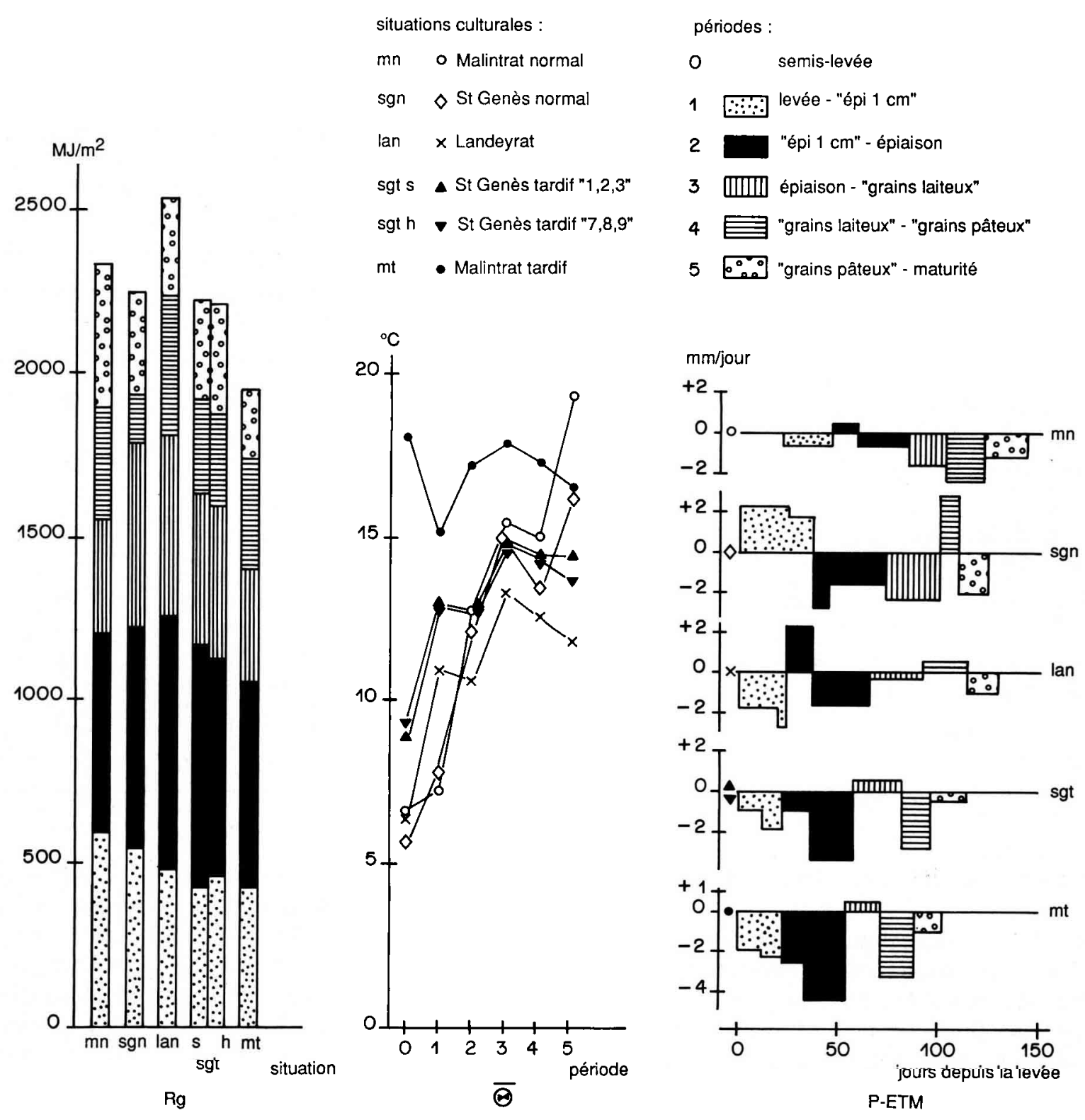

Fig 3. Les climats obtenus dans les différentes situations culturales. Rg: énergie reçue du rayonnement global (mesurée localement par pyranomètre ou calculée à partir de l'insolation locale); $\bar{\theta}:$ température moyenne de l'air (sauf entre semis et levée : température du sol a $-10 \mathrm{~cm}$ ); P-ETM : bilans hydriques climatiques (pluies, évapotranspiration maximale) exprimés en moyenne par jour. L'ETM est estimée à partir d'une ETP Penman locale calée sur une ETP gazon locale (chaque site a comporté des lysimètres conduits en évapotranspiromètres); d'un coefficient cultural pour céréales de printemps (communiqué par Robelin) variant par étapes du cycle végétatif de 0,60 pour un peuplement de jeunes plantules à 0,90 pour un couvert vert fermé en fin de montaison, et décroissant ensuite.

tiel d'utilisation de celui-ci par la croissance des structures et l'entretien de l'équipement métabolique. Entre climats suffisamment différenciés, elle constitue alors un indicateur climatique de disponibilité en assimilats.

Ayant retenu la somme de degrés-jours (ou somme de températures - Durand, 1967) en base $0^{\circ} \mathrm{C}$ (Gallagher et al, 1983) pour indexer le temps, nous exprimons cette intensité de rayon- nement global en énergie reçue par unité de surface au sol et par degré-jour. Le même ratio est dénommé "quotient photothermique» par Fleury et Leterme (1987) pour un autre usage.

Les valeurs obtenues sur notre dispositif par phases du cycle sont présentées au tableau II. Les plus élevées de chaque période proviennent des semis normaux d'altitude. Les écarts peuvent aller du simple au double. 
Tableau II. Intensité moyenne du rayonnement global entre les prélèvements d'étude de la végétation $M J \cdot m^{-2} \cdot{ }^{\circ} C^{-1} \cdot j^{-1}$. Le terme d' cintensité moyenne du rayonnement global» désigne un indicateur climatique d'énergie disponible relativement aux besoins de croissance (voir texte). Il a les dimensions d'une puissance (énergie/temps) par unité de surface, mais on a choisi volontairement une dénomination allusive qui ne l'exprime pas. II ne peut en effet pas avoir le sens qu'on lui prête ici sur moins d'un nycthémère. Ce pas de temps rassemble par définition des périodes très contrastées du point de vue de l'éclairement, le jour et la nuit. Employer un terme dérivé de «flux énergétique par unité de surface» ou «densité de flux de photons», usités par les bioclimatologistes pour quantifier le rayonnement solaire reçu (Rageau, comm pers), pourrait faire croire que notre indicateur est une grandeur physique.

\begin{tabular}{|c|c|c|c|c|c|c|}
\hline $\begin{array}{l}\text { Période et durée } \\
\left({ }^{\circ} \mathrm{C} . j\right)\end{array}$ & $\begin{array}{l}\text { Malintrat } \\
\text { normal }\end{array}$ & $\begin{array}{l}\text { St-Genès } \\
\text { normal }\end{array}$ & Landevrat & $\begin{array}{l}\text { St-Gen } \\
\text { bl } 123\end{array}$ & $\begin{array}{l}\text { s tardif } \\
\text { bl } 789\end{array}$ & $\underset{\text { Malintrat }}{\text { tardif }}$ \\
\hline $\begin{array}{l}\text { Levée - «épi } 1 \mathrm{~cm} \\
\left(200-350^{\circ} \mathrm{Cj}\right)\end{array}$ & 1,73 & 1,87 & 1,92 & 1,58 & 1,55 & 1,29 \\
\hline $\begin{array}{l}\text { Étude tallage «épi } 1 \mathrm{~cm} » \\
\left(120-180^{\circ} \mathrm{Cj}\right)\end{array}$ & 1,13 & 1,33 & 1,92 & 1,53 & 1,54 & 1,08 \\
\hline $\begin{array}{l}\text { Étude tallage-épiaison } \\
\left(290-370^{\circ} \mathrm{Cj}\right)\end{array}$ & 1,29 & 1,60 & 1,64 & 1,55 & 1,55 & 1,19 \\
\hline $\begin{array}{l}\text { Épiaison - «laiteux» } \\
\left(280-370^{\circ} \mathrm{Cj}\right)\end{array}$ & 1,25 & 1,40 & 1,53 & 1,27 & 1,27 & 1,09 \\
\hline $\begin{array}{l}\text { «laiteux» }-\ll \text { pâteux» } \\
\left(120-300^{\circ} \mathrm{Cj}\right)\end{array}$ & 1,18 & 1,21 & 1,54 & 1,40 & 1,40 & 1,14 \\
\hline $\begin{array}{l}\text { "pâteux» - maturité } \\
\left(180-390^{\circ} \mathrm{Cj}\right)\end{array}$ & 1,14 & 1,31 & 1,66 & 1,24 & 1,30 & 0,98 \\
\hline
\end{tabular}

\section{Indicateur de conditions d'humidité}

Les conditions hydriques peuvent être définies par des bilans entre l'évapotranspiration et la somme des précipitations et des réserves en eau du sol. Après une pluie, l'horizon supérieur est d'abord regarni d'eau très disponible (Feodoroff et Baillif, 1969) que les plantes prélèvent préférentiellement (Valancogne et Daudet, 1977). Dans la mesure où les horizons inférieurs ne sont exploités ou remouillés qu'après épuisement ou remplissage de la réserve d'eau de l'horizon supérieur, on peut calculer des bilans sur cette seule couche, particulièrement importante pour l'alimentationn minérale. Sous prairie, les conditions hydriques de l'horizon $0-20 \mathrm{~cm}$ sont déterminantes pour l'alimentation azotée (Lemaire et Denoix, 1987). Sous culture, il faut prendre en compte les profondeurs d'enfouissement des résidus et des fumures. C'est alors plutôt la couche $0-30 \mathrm{~cm}$ qui doit être considérée comme horizon fournisseur d'éléments nutritifs. On peut l'appeler couche arable.

L'humidité de la couche arable est très variable. Pour comparer des situations entre elles, il nous a semblé intéressant de calculer par pé- riode la fraction de la demande évaporative qui pouvait être satisfaite par les ressources de cet horizon supérieur. C'est un estimateur du temps pendant lequel la partie principale de l'enracinement a pu fonctionner dans des conditions d'humidité acceptables. On propose ainsi un indicateur dénommé FERA et variant de 0 à 1 . On le calcule en divisant l'évapotranspiration maximale de la période par les pluies augmentées des réserves dans l'horizon $0-30 \mathrm{~cm}$ en début de période. Quand les ressources dépassent la demande, le rapport est plafonné à 1 et l'excédent reporté. Les réserves étant faibles, elles comptent très peu dans le bilan pour les périodes de pleine végétation. Les erreurs considérables dues aux reports successifs de soldes de bilan sont donc ici de peu d'importance. L'indicateur FERA est étroitement corrélé au bilan purement climatique P-ETM (Lafarge, 1991).

Le tableau III donne les valeurs de l'indicateur FERA sur notre dispositif pour les mêmes périodes que le tableau II. Les semis tardifs ont rencontré des conditions hydriques plus contrastées au cours du cycle que les semis normaux. lls ont subi une sécheresse marquée en montaison. 


\section{LE «SUIVI»}

\section{Rendements et caractères de végétation pertinents pour l'étude du climat}

Les rendements sont généralement très faibles en montagne, mais les cultures conduites avec les mêmes soins qu'en plaine y sont aussi productives. Les $100 \mathrm{q} / \mathrm{ha}$ ont été atteints plusieurs fois en essais d'automne (Triboï et al, 1985; Gate et al, 1990). Sauf sécheresse ou acidité excessives, les comparaisons de champs de production situés à diverses altitudes montrent que les écarts de rendements agricoles découlent des différences d'interventions techniques et de fertilisation que subissent les cultures (Lafarge, 1983).

Nos résultats permettent de faire une constatation analogue (fig 4) : les dates de semis et les conduites sont importantes, et les meilleurs traitements ont des rendements équivalents en montagne et en plaine. Le tableau IV montre pourtant que ces rendements sont obtenus de façons assez différentes.

C'est une verse grave qui a empêché Malintrat «normal FN» d'être aussi productif que les autres. L'occurrence de ce phénomène révèle les différences de conditions ou de comportement des plantes entre situations culturales. Le traitement FN de Malintrat normal a subi à 780 épis/ $\mathrm{m}^{2}$ une verse générale précoce. Son homologue de Landeyrat, qui n'a été affecté qu'à maturité et par petits foyers, en porte 100 de plus.

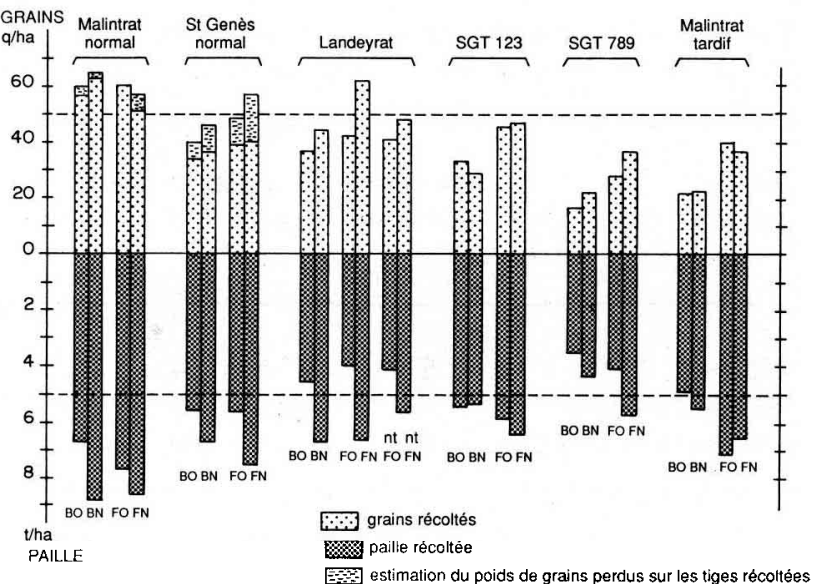

Fig 4. Rendements moyens en grains et paille par traitement élémentaire exprimés aux normes agricoles $(15 \%$ d'eau). Les grains perdus l'ont été par attaques d'oiseaux en fin de végétation. Leur poids est estimé à partir du poids moyen du grain sur le traitement et d'un comptage des insertions de grains où les glumelles manquent.

Au long du cycle, les comportements des plantes ont différé entre situations. Plus que le rendement ou même ses composantes, c'est la succession dans le temps des états de développement et de croissance qui peut traduire les effets du climat.

\section{Le programme de mesures}

Nous avons cherché à reconstituer la formation du nombre de tiges fertiles, depuis le déroule-

Tableau III. Valeurs de l'indicateur «FERA» pour les périodes délimitées par les prélèvements de végétation. L'indicateur que nous dénommons «FERA" représente la fraction de l'ETM couverte par la ressource de la couche arable (voir texte). Les prélèvements ne sont pas tous caractérisés par un stade phénologique moyen; le prélèvement pour étude du tallage est défini dans le texte.

\begin{tabular}{|c|c|c|c|c|c|c|}
\hline Période & $\begin{array}{c}\text { Malintrat } \\
\text { normal }\end{array}$ & $\begin{array}{c}\text { St-Genès } \\
\text { normal }\end{array}$ & Landeyrat & $\begin{array}{l}\text { St-Genè } \\
\text { bl } 123\end{array}$ & $\begin{array}{l}\text { s tardif } \\
\text { bl } 789\end{array}$ & $\begin{array}{c}\text { Malintrat } \\
\text { tardif }\end{array}$ \\
\hline Levée - “épi $1 \mathrm{~cm}$ » & 1 & 1 & 0,8 & 0,9 & 1 & 0,6 \\
\hline $\begin{array}{l}\text { "épi } 1 \mathrm{~cm} \text { - } \\
\text { "étude tallage" }\end{array}$ & 1 & 1 & 1 & 0,7 & 0,9 & 0,3 \\
\hline $\begin{array}{l}\text { "étude tallage" - } \\
\text { "épiaison" }\end{array}$ & 1 & 0,7 & 0,8 & 0,1 & 0,1 & 0,1 \\
\hline «épiaison» - «laiteux» & 0,7 & 0,5 & 0,9 & 1 & 1 & 1 \\
\hline "laiteux" - "pâteux" & 0,3 & 1 & 1 & 0,4 & 0,4 & 0,2 \\
\hline "pâteux" - maturité & 0,6 & 0,9 & 0,9 & 0,9 & 0,9 & 0,6 \\
\hline
\end{tabular}


Tableau IV. Caractéristiques du rendement du meilleur traitement fertilisé en semis normal dans chaque site. (") $\mathrm{HI}=$ poids de grains en \% du poids du total des parties aériennes à la même humidité.

\begin{tabular}{lccccc}
\hline $\begin{array}{l}\text { Site et } \\
\text { altitude }\end{array}$ & $\begin{array}{c}\text { Traitement } \\
\text { cultural }\end{array}$ & $\begin{array}{c}\text { Rendement } \\
(q / \text { ha à 15\% d'eau) }\end{array}$ & $\begin{array}{c}\text { Indice de récolte } \\
H I\left(^{*}\right)\end{array}$ & $\begin{array}{c}\text { Nombre d'épis } \\
\text { par m² } \\
\text { (tardillons exclus) }\end{array}$ & $\begin{array}{c}\text { Nombre de grains } \\
\text { par épi } \\
\text { (tardillons exclus) }\end{array}$ \\
\hline & & & & & \\
Malintrat 320 m & BN & 64 & $42 \%$ & 530 & 23,8 \\
St-Genès $880 \mathrm{~m}$ & FN & 57 & $43 \%$ & 636 & 20,1 \\
Landeyrat 1 120 m & FN & 62 & $48 \%$ & 879 & 15,2 \\
\hline
\end{tabular}

ment du tallage herbacé jusqu'au nombre d'épis avec leurs âges relatifs en fin de culture. Sur un prélèvement effectué en début de montaison, quand le tallage paraît arrêté, nous avons compté plante à plante les feuilles sur les différentes tiges présentes. Si le modèle de Masle (MasleMeynard et Sébillotte, 1981) s'applique, ce qu'on peut espérer (Kirby et al, 1985), on peut dater a posteriori le début et la fin du tallage de chaque plante d'un échantillon. En fin de végétation, vers le stade grain pâteux, les tiges présentes sont réparties en catégories dont les effectifs peuvent être comparés à différents regroupements de tiges à l'arrêt du tallage. Les prélèvements étant destructifs, il s'agit de comparaisons d'effectifs entre échantillons différents, dont on peut cependant tester la représentativité à partir de caractères réputés invariables.

La formation du nombre de grains par épi est décrite rétrospectivement à partir du prélèvement stade pâteux évoqué ci-dessus. Sur chaque épi caractérisé par l'allongement de sa tige, son ordre et son rang sur le pied et sa tardiveté, nous avons dénombré les fleurettes avortées en cours de croissance, les fleurs normales vides de grain, les grains et les insertions dépourvues de glumelles signalant un grain formé puis enlevé, par exemple par un oiseau.

Pour suivre les gains de poids sec, les parties aériennes ont été pesées à 4 stades; “épi $1 \mathrm{~cm}$ ", épiaison, grains laiteux et maturité.

\section{CONSÉQUENCES DE CONDITIONS NATURELLES DIFFÉRENCIÉES}

\section{Résultats obtenus sur le dispositif}

Les états successifs de la végétation seront décrits et analysés dans des articles ultérieurs. Nous avons insisté sur l'analyse de la variabilité interne à chaque traitement élémentaire, mais nous avons aussi cherché au niveau de l'ensemble du dispositif une explication commune aux caractères des différents peuplements. Les indicateurs proposés ci-dessus se sont avérés statistiquement utiles pour ces analyses globales. On peut citer par exemple (Lafarge, 1991):

- l'indicateur d'humidité dans la couche arable (FERA) intervient significativement dans les changements de niveau de nutrition azotée entre prélèvements;

- l'intensité moyenne du rayonnement global est un estimateur majeur du taux moyen de transformation des ébauches florales en fleurs normales puis de mise à grains de celles-ci.

\section{Variation de la variabilité}

Le tableau $V$ donne les coefficients de variation résiduels obtenus pour quelques caractères dans chaque situation culturale. Ils résultent chacun d'une analyse de variance à 3 facteurs croisés (2 facteurs expérimentés et l'effet bloc). La variablité - qui diffère bien sûr selon les caractères - varie pour un même caractère entre situations culturales. Cette variation est un indice des différences entre les écosystèmes cultivés obtenus avec un même cultivar quand le milieu change suffisamment.

Rarement envisagée, cette situation pourrait bien être générale dès que les conditions varient en milieu naturel (Krajewski, 1990). Elle interdit d'admettre l'égalité des variances sur l'ensemble d'un dispositif multilocal.

\section{Implications statistiques}

Les tests effectués à l'issue d'une analyse de variance et en comparaison de moyennes supposent la normalité des distributions et l'égalité des variances entre les différentes cellules élémen- 
Tableau V. Coefficients de variation résiduelle des mesures de 4 caractères de végétation pour chaque dispositif local. Dans chaque situation et pour Landeyrat "parcelles traitées", l'analyse de variance portait sur les facteurs densité de semis, fumure azotée et bloc. Pour Landeyrat «toutes fortes densités», le facteur «traitement phytosanitaire» se substitue à la densité de semis.

\begin{tabular}{|c|c|c|c|c|c|c|c|c|}
\hline & $\begin{array}{c}\text { Surface de } \\
\text { la placette } \\
\text { de mesure }\left(m^{2}\right)\end{array}$ & $\begin{array}{c}\text { Malintrat } \\
\text { normal }\end{array}$ & $\begin{array}{c}\text { St-Genès } \\
\text { normal }\end{array}$ & $\begin{array}{l}\text { Landeyrat } \\
\text { parcelles } \\
\text { traitées }\end{array}$ & $\begin{array}{l}\text { Landeyrat } \\
\text { toutes fortes } \\
\text { densités }\end{array}$ & $\begin{array}{c}\text { St-Genès } \\
\text { tardif } \\
« 1,2,3 »\end{array}$ & $\begin{array}{c}\text { St-Genès } \\
\text { tardif } \\
\ll 7,8,9 »\end{array}$ & $\begin{array}{c}\text { Malintrat } \\
\text { tardif }\end{array}$ \\
\hline Nombre de blocs & & 6 & 6 & 6 & 6 & 3 & 3 & 6 \\
\hline $\begin{array}{l}\text { Poids d'orge } \\
\text { pr «épi } 1 \mathrm{~cm} \text { " }\end{array}$ & 0,26 & $0,6 \%$ & $13,4 \%$ & $21,8 \%$ & $22,7 \%$ & $26,2 \%$ & $21,0 \%$ & $35,1 \%$ \\
\hline $\begin{array}{l}\text { Poids d'orge } \\
\text { pr «laiteux» }\end{array}$ & 0,26 & $11,3 \% *$ & $16,1 \%$ & $22,3 \%$ & $11,2 \% *$ & $10,8 \%$ & $21,4 \%$ & $29,9 \%$ \\
\hline $\begin{array}{l}\text { Nombre d'épis } \\
\text { pr «pâteux» }\end{array}$ & 1,50 & $19,0 \%$ & $11,8 \%$ & $12,2 \%$ * & $11,8 \%$ & $4,0 \%$ & $4,3 \%$ & $8,6 \%$ \\
\hline $\begin{array}{l}\text { Poids moyen } \\
\text { d'un grain } \\
\text { d'épi normal }\end{array}$ & 0,26 & $2,5 \% *$ & $6,5 \%$ & $1,5 \%$ & $5,2 \%$ & $5,8 \% *$ & $5,3 \%$ & $3,8 \%$ \\
\hline
\end{tabular}

" Effet «bloc» significatif retranché de la variance résiduelle.

taires du dispositif. Le défaut de normalité a peu d'incidence sur le résultat; il n'en est pas de même pour l'inégalité des variances, surtout si les effectifs compris dans chaque cellule élémentaire sont inégaux (Dagnélie, 1984). Tant que les populations sont définies par un seul facteur contrôlé, l'analyse d'un modèle linéaire général (comme dans la procédure GLM du logiciel SAS) reste fiable. Ce n'est plus le cas pour des dispositifs à plusieurs facteurs croisés, qualifiés alors de non orthogonaux (Dagnélie, 1984).

Dans notre étude, le nombre de répétitions varie dès qu'on veut comparer les situations culturales et dans les nombreux cas où les individus ne sont plus les placettes mais les plantes ou les tiges d'un prélèvement par placette. Pour travailler dans ces conditions, on a utilisé la méthode de Gaylor et al (1970), disponible dans le logiciel Amance (Bachacou et al, 1981). Elle permet l'analyse de dispositifs non orthogonaux à plusieurs facteurs. Sur la base d'une matrice des poids des individus participant aux différentes cellules élémentaires du dispositif et aux interactions, on calcule un $F$ ajusté pour un premier facteur, non ajusté pour les autres. Les calculs sont ensuite répétés avec un nouvel ajustement pour un deuxième facteur, et ainsi de suite. Appliquée à un dispositif orthogonal, cette méthode donne la même valeur au $F$ ajusté et non ajusté et des résultats identiques à chaque répétition des calculs.
Grâce à cet outil, on a pu utiliser l'analyse de variance dans des investigations d'enquête. Les conditions et les états réellement obtenus suggèrent en effet de nouvelles structurations du dispositif d'ensemble; par exemple, par des niveaux d'âge des plantes à l'enclenchement du développement reproducteur, ou par des niveaux de sécheresse à telle ou telle phase du cycle. Restructuré a posteriori sur la base de tels facteurs, un dispositif n'a pratiquement aucune chance d'être orthogonal. II est pourtant aussi important d'analyser les effets liés à ces états que ceux découlant directement du plan d'expérience.

\section{CONCLUSION}

Dispositifs expérimentaux, enquêtes avec suivi, indicateurs d'états et méthodes statistiques acceptant des différences de variabilité constituent des pièces complémentaires pour l'analyse des effets de climats naturels différents sur un matériel végétal déterminé.

Les résultats montrent que le rendement peut être un mauvais indicateur des effets du climat. L'analyse des états successifs de la végétation est nécessaire. On arrive ainsi à une étude écologique de culture. 


\section{RÉFÉRENCES}

Aspinall $D$ (1961) The control of tillering in the barley plant. 1. The pattern of tillering and its relation to nutrient supply. Aust J Biol Sci 14, 493-505

Bachacou J, Masson JP, Millier C (1981) Manuel de la programmathèque statistique «Amance 81 ». INRA Biométrie, 54280 Champenoux

Bazin G, Larrère GR (1983) Du système agropastoral à la spécialisation laitière. In: Système agraire et pratiques paysannes dans les monts Dômes ( $G$ Bazin et al) INRA, Versailles, 1-153

Biscoe PV, Clark JA, Gregson K, McGowan M, Monteith JL, Scott RK (1975) Barley and its environment. 1. Theory and practice. $J$ App/ Ecol 12, 227257

Boiffin J, Sébillotte M (1975) Influence des conditions écologiques et de l'histoire culturale sur le rendement du maïs-grain. Analyse de 8 campagnes sur un essai de longue durée. Ann Agron 26, 555-591

Cabelguenne M, Charpenteau JL, Jones CA, Marty JR, Rellier JP (1986) Conduite des systèmes de grande culture et prévision des rendements : tentative de modélisation. 2. Étalonnage du modèle : résultats et perspectives. $C R$ Seances Acad Agric Fr 72, 125-132

Cabelguenne $M$, Jones CA, Marty JR, Quinones H (1988) Contribution à l'étude des rotations culturales : tentative d'utilisation d'un modèle. Agronomie 8, 549-556

Chamberlin RJ, Insomphun S (1982) Growth and development of upland rice at three altitudes in the highlands of northern Thailand. Exp Agric 18, 363373

Charpenteau JL, Jones CA, Marty JR, Rellier JP, Williams JR (1986) Conduite des systèmes de grande culture et prévision des rendements : tentative de modélisation. 1. Choix et construction du modèle. C R Seances Acad Agric Fr 72, 118-124

Collier D (1949) Contribution à l'étude des terres noires de la Limagne d'Auvergne : constitution et genèse. Ann Agron (ancienne série) 1949, 1-77

Dagnélie P (1984) Théories et méthodes statistiques. Applications agronomiques. Presses agronomiques, Gembloux (Belgique), tome 2

Durand R (1967) Action de la température et du rayonnement sur la croissance. Ann Physiol Vég 9, 5-27

Ellis RP, Brown $\mathrm{J}$ (1986) Yield in spring barley at contrasting sites in England and Scotland. Ann Appl Biol 109, 613-617

Evans LT (1963) Extrapolation from controlled environments to the field. In: Environmental control of plant growth (LT Evans, ed) Acad Press, New York, 421-437

Feodoroff A, Baillif JL (1969) Étude de l'infiltration de la pluie in situ à l'aide de tensiomètres. Ann Agron $20,475-504$
Fleury A, Leterme P (1987) Conditions d'utilisation du quotient photothermique pour le diagnostic cultural, CR Seances Acad Agric Fr 73, 37-43

Fuchs W (1984) Einfluss von Jahreswitterung, Standort und Stickstoffdüngung auf Ertrag und Brauqualität der Sommergerste. Arch Acker-Pflanzenbau Bodenkd 28, 45-50

Gallagher JN, Biscoe PV, Scott RK (1976) Barley and its environment. 6. Growth and development in relation to yield. J Appl Ecol 13, 563-583

Gallagher JN, Biscoe PV, Dennis-Jones R (1983) Environmental influences on the development, growth and yield of barley. In: Barley, production and marketing (GM Wright, RB Wynn-Williams, eds) Agron Soc N Z Spec Publ, 21-49

Gate P, de Mijolla P, Laroche G, Nouailles (1990) Comportement et adaptation des céréales à paille en montagne de l'est du Massif Central. Perspect Agric 147, 51-64

Gaylor DW, Lucas HL, Anderson RL (1970) Calculation of expected mean squares by the abbreviated Doolittle and square root methods. Biometrics 26, 641-656

Gosse G, Varlet-Grancher L, Bonhomme R, Chartier M, Allirand JM, Lemaire G (1986) Production maximale de matière sèche et rayonnement solaire intercepté par un couvert végétal. Agronomie 6, 47. 56

Gras R, Benoît M, Deffontaines JP, Duru M, Lafarge M, Langlet A, Osty PL (1989) Le fait technique en agronomie; actiotí agricole, concepts et méthodes d'étude. INRA et L'Harmattan, Paris

Greenwood DJ, Neeteson JJ, Draycott A (1986) Quantitative relationships for the dependance of growth rate of arable crops on their nitrogen content, dry weight and aerial environment. Plant Soil 91, 281301

Hétier JM (1971) Caractérisation et répartition de quelques sols sur roches volcaniques du Massif Central (région de Menet, Cantal). Sci Sol 1971 n², 51-82

Hough MN (1975) Meteorological models for the effects of weather on barley development and yield. In: Progress in biometeorology, div $C$ : progress in plant biometeorology, vol 1, 240-251

Hunter RF, Grant SA (1971) The effect of altitude on grass growth in east Scotland. $J$ Appl Ecol 8, 1-19

Kirby EJM, Faris DG (1970) Plant population induced growth correlations in the barley plant main shoot and possible hormonal mechanisms. $J$ Exp Bot 21, 787-798

Kirby EJM, Appleyard M (1981) Cereal development guide. Cereal Unit, National agricultural center, Stoneleigh, UK

Kirby EJM, Appleyard M, Fellowes G (1985) Leaf emergence and tillering in barley and wheat. Agronomie 5, 193-200

Kobold F (1974) Zur Ertragsbildung von Winterweizen und Sommergerste in unterschiedlichen Höhenlage. Arch Acker-Pflanzenbau Bodenkd 18, 407 414 
Krajewski $P(1990)$ Heterogeneity of variance in field experiments; some causes and practical limitations. $J$ Agric Sci Camb 115, 83-94

Lafarge $M$ (1983) Approche des facteurs et conditions du rendement des céréales en altitude. $C R$ Séances Acad Agric Fr 69, 917-928

Lafarge $M$ (1987) Principaux effets du climat sur la croissance et le développement des céréales en altitude dans le Massif Central. In: Agrométéorologie des régions de moyenne montagne. "Les Colloques de l'INRA" n*39, 287-302

Lafarge M (1991) Effets de l'altitude sur le tallage, la fertilité d'épi et la croissance d'une orge de printemps cultivée en peuplements. Thèse Docteur INAPG, Paris

Lafarge M, André V, Carette B, Pravin C (1982) Limitation de productivité des céréales par le climat d'altitude et par les techniques compatibles avec les systèmes de culture montagnards. C R contrat DGRST $n^{\circ}$ 77-7 1955-1956

Lawlor JG, Day W, Johnston AE, Legg BJ, Parkinson KJ (1981) Growth of spring barley under drought: crop development, photosynthesis, dry matter accumulation and nutrient content. J Agric Sci Camb 96, 167-186

Lemaire G, Salette J (1984) Relation entre dynamique de croissance et dynamique de prélèvement d'azote pour un peuplement de graminées fourragères. 1 . Étude de l'effet du milieu. Agronomie 4, 423-430

Lemaire G, Denoix A (1987) Croissance estivale en matière sèche de peuplements de fétuque élevée et de dactyle dans l'ouest de la France. 2. Interaction entre les niveaux d'alimentation hydrique et de nutrition azotée. Agronomie 7, 381-389

Masle-Meynard J (1981) Élaboration du nombre d'épis d'un peuplement de blé d'hiver en situation de compétition pour l'azote. 1. Mise en évidence d'un stade critique pour la montée d'une talle. Agronomie 1, 623-632

Masle $J$ (1985) Competition among tillers in winter wheat: consequences for growth and development of the crop. In: Wheat growth and modelling ( $W$ Day, RK Atkin, eds) Plenum press, New York, NATO-ASI serie A life science, vol 86, 33-54

Masle-Meynard J, Sébillotte M (1981) Étude de l'hétérogénéité d'un peuplement de blé d'hiver. 2. Origine des différentes catégories d'individus du peuplement; éléments de description de sa structure. Agronomie 1, 217-224

Paleg LG, Aspinall D (1966) Effects of day length and light intensity on growth of barley. 5. Response by plants in the field to night interruption. Aust $J$ Biol Sci 19, 719-731

Prince SD (1976) The effect of climate on grain development in barley at an upland site. New Phytol 76, 377-389

Rellier JP (1980) Analyse statistique de la variabilité des rendements sur le dispositif expérimental avec rotations d'Auzeville. In: Méthodologie d'étude des systèmes de culture (séminaire Toulouse mai 1980) CEE-Agrimed, INRA, Toulouse, 175-189

Rémy JC, Hébert J (1977) Le devenir des engrais azotés dans le sol. C R Seances Acad Agric Fr 63, 700-714

Riggs TJ (1986) Collaborative spring barley trials in Europe 1980-1982. Analysis of grain yield. Z Pflanzenzuecht 96, 289-303

Stegemann K, Enke H, Bahn E (1987) Untersuchungen zur Häufigkeitsverteilung von Wintergerstenerträgen aus den Jahren 1920 bis 1981 in Abhängigkeit von der Dauer der Vorwinterentwicklung, der Witterung im Winterhalbjahr und der Dauer der generative Phase. Arch Acker-Pflanzenbau Bodenkd 31, 205-212

Triboï E, Gachon L, Morizet J (1985) Les potentialités de production du blé d'hiver en moyenne montagne du Massif Central. Agronomie 5, 135-142

Valancogne C, Daudet FA (1977) Étude in situ du potentiel hydrique sous une culture de maîs à l'aide d'un système automatique de mesure à micropsychromètres à effet Peltier. Ann Agron 28, 137157

Van Diepen CA, Wolf J, Van Keulen H, Rappoldt C (1989) WOFOST: a simulation model of crop production. Soil Use Manage 5, 16-24

Vincent A (1986) Économie de la plante : importance de l'installation et de l'activité des racines. In : L'élaboration du rendement des cultures céréalières (colloque franco-roumain, L Gachon, ed) INRA, Paris, 31-55 